\title{
The scattering amplitude of ultracold atoms near the p-wave magnetic Feshbach Resonance
}

\author{
Peng Zhang, ${ }^{1,2}$ Pascal Naidon, ${ }^{1}$ and Masahito Ueda ${ }^{1,3}$ \\ ${ }^{1}$ ERATO, JST, Macroscopic Quantum Control Project, Hongo, Bunkyo-Ku, Tokyo 113-8656, Janan \\ ${ }^{2}$ Department of Physics, Renmin University of China, Beijing, 100190, China \\ ${ }^{3}$ Department of Physics, University of Tokyo, Hongo, Bunkyo-Ku, Tokyo 113-8656, Janan
}

\begin{abstract}
Most of the current theories on the p-wave superfluid in cold atomic gases are based on the effective-range theory for the two-body scattering, where the low energy p-wave scattering amplitude $f_{1}(k)$ is given by $f_{1}(k)=-1 /\left[i k+1 /\left(\mathcal{V} k^{2}\right)+1 / \mathcal{R}\right]$, where $k$ is the incident momentum, and $\mathcal{V}$ and $\mathcal{R}$ are the $k$-independent scattering volume and effective-range, respectively. However, due to the long-range nature of the van der Waals interaction between two colliding ultracold atoms, the p-wave scattering amplitude of the two atoms is not described by the effective-range theory $[1,2]$. In this paper we provide an explicit calculation for the p-wave scattering of two ultracold atoms near the p-wave magnetic Feshbach resonance (PMFR). We show that the low energy pwave scattering amplitude in the presence of PMFR takes the form $f_{1}(k)=-1 /\left[i k+1 /\left(\mathcal{V}^{\text {eff }} k^{2}\right)+\right.$ $\left.1 /\left(\mathcal{S}^{\text {eff }} k\right)+1 / \mathcal{R}^{\text {eff }}\right]$ where $\mathcal{V}^{\text {eff }}, \mathcal{S}^{\text {eff }}$ and $\mathcal{R}^{\text {eff }}$ are $k$-dependent parameters. Based on this result, we show sufficient conditions for the effective range theory to be a good approximation of the exact scattering amplitude. Using these conditions we show that the effective-range theory is a good approximation for the p-wave scattering in the ultracold gases of ${ }^{6} \mathrm{Li}$ and ${ }^{40} \mathrm{~K}$ when the scattering volume is enhanced by the resonance.
\end{abstract}

PACS numbers:

\section{INTRODUCTION}

Recently, ultracold atomic gases with strong p-wave interaction have attracted broad interest both experimentally [3-15] and theoretically [16 65]. The p-wave magnetic Feshbach resonances, which can generate tunable p-wave ineratomic interactions, have been observed in the cold gases of ${ }^{40} \mathrm{~K}[3-5],{ }^{6} \mathrm{Li}[8,9],{ }^{6} \mathrm{Li}^{-}{ }^{87} \mathrm{Rb}$ mixture [13], ${ }^{6} \mathrm{Li}-{ }^{40} \mathrm{~K}$ mixture [14], and ${ }^{40} \mathrm{~K}-{ }^{87} \mathrm{Rb}$ mixture [15]. The p-wave Feshbach molecules have also been created and studied in the gases of ${ }^{40} \mathrm{~K}[6,7]$ ] and ${ }^{6} \mathrm{Li}$ [8, 10 12]. These experimental achievements stimulate theoretical researches on the quantum superfluid in ultracold atomic gases with strong p-wave interactions 16 52], as well as the relevant few-body problems 52 65].

Until now, most theories of ultracold atomic gases with strong p-wave interactions [35 38, 40 42, 52 57] are based on the low energy expansion of the p-wave scattering amplitude $f_{1}(k)$ given by the effective-range theory 66

$$
f_{1}(k)=-\frac{1}{i k+\frac{1}{\mathcal{V} k^{2}}+\frac{1}{\mathcal{R}}}
$$

Here $\vec{k}$ is the relative momentum of the two atoms; $\mathcal{V}$ is the scattering volume and $\mathcal{R}$ is the effective-range. The effective-range theory for the scattering amplitude is used in both theories of p-wave atomic superfluids 35 38, 40 42, 52 and related few-body problems 52 57]. In particular, the separable two-body potential 37] $V\left(k, k^{\prime}\right)=\lambda_{1} w(k) w\left(k^{\prime}\right)$ used in the many-body Hamiltonian for the p-wave atomic superfluid is derived directly from low energy expansion (1) of the scattering amplitude.
However, the effective-range theory is correct only for the short range potentials (e.g., Yukawa potential) 66] which decays faster than any power function $r^{-\gamma}$ in the large interatomic distance limit $r \rightarrow \infty$. Here $\vec{r}$ is the relative coordinate between two atoms. For a realistic interaction between two cold atoms, which is described by a long-range potential dominated by the van der Waals term $-\hbar^{2} \beta_{6}^{4} /\left(r^{6} m\right)$ in the limit $r \rightarrow \infty$. Here $m$ is the single-atom mass, $\hbar$ is the Plank constant and $\beta_{6}$ is the van der Waals length. Due to the long-range van der Waals potential, the effective-range theory and the lowenergy expansion (11) of the p-wave scattering amplitude is not applicable any longer [1, 2].

In the presence of a p-wave magnetic Feshbach resonance (PMFR) in the ultracold gases of polarized fermionic atoms, the p-wave scattering amplitude of the atomic collision is contributed by both the background potential in the open channel and the bound state in the closed channel. The long-range nature of the background potential makes the final scattering amplitude to be inconsistent with the effective-range theory.

Therefore, it is essential to investigate the condition under which the effective-range theory (1) can be used as an approximation of the exact p-wave scattering amplitude under a PMFR. If the effective-range theory provides a good approximation of the scattering amplitude, then the previous theories on p-wave superfluid would be applicable; nevertheless, if the exact scattering amplitude is found to be significantly different with the one in Eq. (1), then the previous theories should be modified. Especially, the separable two-body potential cannot be used any more.

The low-energy p-wave scattering amplitude near PMFR has been investigated in Refs. [53, 55]. How- 
ever, these studies are based on simplified models of the atomic interaction, e.g., zero background potential [53] or a separable background potential that decays exponentially in the momentum space [55]. The long-range van der Waals potential is not taken into account in either case. Due to these simplifications, the scattering amplitudes given in Refs. [53, 55] automatically have the form of Eq. (1), and cannot be used to judge the applicability of the effective-range theory.

In this paper, based on the realistic long-range interatomic potential, we provide an explicit calculation for the low-energy p-wave scattering amplitude of two spin polarized fermonic atoms near a PMFR, and then discuss the condition under which the effective-range theory can be used as a good approximation. We get sufficient conditions for the effective-range theory, and show that for the ultracold gases of of ${ }^{6} \mathrm{Li}$ and ${ }^{40} \mathrm{~K}$ with the Fermi temperature of the order $1 \mu \mathrm{K}$, the effective-range theory can be used as a good approximation in the resonance regime where the scattering volume is enhanced

\section{A. Main results} lows lows.

In this work we first calculate the exact expression of the low energy p-wave scattering amplitude with a PMFR. We prove that the scattering amplitude can be expressed as

$$
\begin{aligned}
& f_{1 m_{z}}(k) \\
= & -\frac{1}{i k+\frac{1}{\mathcal{V}^{\text {eff }}\left(k ; B ; m_{z}\right) k^{2}}+\frac{1}{\mathcal{S}^{\text {eff }}\left(k ; B ; m_{z}\right) k}+\frac{1}{\mathcal{R}^{\text {eff }}\left(k ; B ; m_{z}\right)}} .
\end{aligned}
$$

with $B$ the strength of the magnetic field applied along the $z$ axis. Here $m_{z}$ is the $z$-component of the angular momentum; $\mathcal{V}^{\text {eff }}, \mathcal{S}^{\text {eff }}$ and $\mathcal{R}^{\text {eff }}$ are $k$-dependent scattering parameters. We obtain the general expressions for them. It is pointed out that, the denominator of $f_{1 m_{z}}(k)$ cannot be expressed as a Laurent series with $k$ independent coefficients because $\mathcal{V}^{\text {eff }}, \mathcal{S}^{\text {eff }}$ and $\mathcal{R}^{\text {eff }}$ are not analytical functions of $k$.

Equation (2) shows that, in the presence of a PMFR the inconsistency of the scattering amplitude with the effective-range theory is displayed in a more complicated manner. The low-energy p-wave scattering amplitude in Eq. (2) is different from the one obtained from the standard effective-range theory in the following two senses:

1. The scattering parameters $\left(\mathcal{V}^{\text {eff }}, \mathcal{S}^{\text {eff }}, \mathcal{R}^{\text {eff }}\right)$ depend on the incident momentum $k$.

2. The term $1 /\left[\mathcal{S}^{\text {eff }}\left(k ; B ; m_{z}\right) k\right]$ cannot be included in the effective-range theory.

After obtaining the p-wave scattering amplitude under a PMFR, we discuss the applicability of the effective rang theory as an approximation of the scattering amplitude (2). We find that, in the BEC side of the PMFR where
$\mathcal{V}^{\text {eff }}$ and $\mathcal{R}^{\text {eff }}$ have the same sign, sufficient conditions for the validity of effective-range theory are $r_{1}, r_{2}<<1$. In the BCS side of the resonance, the sufficient conditions become $r_{1}, r_{2}, r_{3}<<1$. Here $r_{1}, r_{2}$ and $r_{3}$ are defined in Eqs. (38), (33) and (42). If these conditions are satisfied, the scattering amplitude of our system can be approximated as

$$
f_{1 m_{z}}(k) \approx-\frac{1}{i k+\frac{1}{\mathcal{V}^{\text {eff }}\left(0 ; B ; m_{z}\right) k^{2}}+\frac{1}{\mathcal{R}^{\text {eff }}\left(0 ; B ; m_{z}\right)}}
$$

which has the same form as Eq. (1) derived by the effective-range theory.

Qualitatively speaking, the above sufficient conditions means that we can use the effective-range theory if the fermonic momentum of the cold gas is low enough, the magnetic field is tuned close enough to the resonance point and the background scattering potential in the open channel is far away from the zero-energy shape resonance point. For the realistic cold gases of Fermi atoms, if the background scattering is far away from the shape resonance, the effective range theory can usually be used in the total region where the $p$-wave interaction is negligible.

The paper is organized as follows. In Sec. II, we calculate the p-wave scattering amplitude near a PMFR, and obtain the low energy expansion in (2). The parameters $\left(\mathcal{V}^{\text {eff }}, \mathcal{S}^{\text {eff }}, \mathcal{R}^{\text {eff }}\right)$ are expressed in terms of the background scattering parameters and the magnetic field. In Sec. III we discuss the range of applicability of the effectiverange theory. We show that, under some simple sufficient conditions $r_{1}, r_{2}<<1$ or $r_{1}, r_{2}, r_{3}<<1$, the scattering amplitude given by the effective-range theory is a good approximation for the exact one obtained in Sec. II. We further show that these conditions are well satisfied in the cold gases of ${ }^{40} \mathrm{~K}$ and ${ }^{6} \mathrm{Li}$ when the scattering volume is enhanced by a PMFR, and then the previous results based on the effective-range theory are applicable for these systems. In Sec. IV there are some conclusion and discussions. We describe some detail of our calculations in the appendixes.

\section{LOW-ENERGY SCATTERING AMPLITUDE NEAR THE P-WAVE FESHBACH RESONANCE}

\section{A. p-wave phase shifts with PMFR}

In this section we calculate the p-wave scattering amplitude in presence of a PMFR induced by a magnetic field along the $z$ direction. We begin with the twochannel Hamiltonian for the relative motion of two atoms (Fig. 1):

$$
H=\left(\begin{array}{cc}
\hat{T}+V^{(\mathrm{bg})}(r) & W(r) \\
W(r) & \hat{T}+V^{(\mathrm{cl})}(\vec{r})+\varepsilon(B)
\end{array}\right),
$$




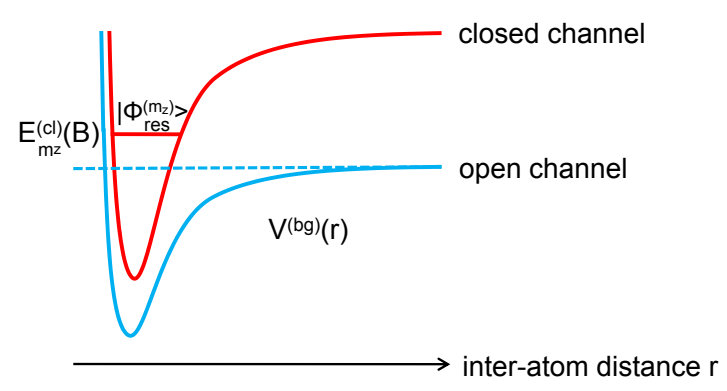

FIG. 1: (color online) The two channel model of the p-wave Feshbach resonance.

where $\hat{T}$ is the kinetic energy of relative motion, $V^{(\mathrm{bg})}(r)$ is the background scattering potential in the open channel, $W(r)$ is the coupling between the open and closed channel, and $V^{(\mathrm{cl})}(\vec{r})$ is the interaction potential in the close channel, that has a $B$-dependent positive threshold $\varepsilon(B)$. In this paper, for simplicity, we assume that the background potential $V^{\mathrm{bg}}(r)$ is independent on the direction of $\vec{r}$ and invariant under the $\mathrm{SO}(3)$ rotation. We further assume that, in the closed channel there are only three bare p-wave bound states $\left|\phi_{\text {res }}^{\left(m_{z}\right)}\right\rangle$ which are near resonance with the threshold of the open channel. Here $m_{z}=0, \pm 1$ is the angular momentum along the $z$ axis. The self energy $E_{m_{z}}^{(\mathrm{cl})}(B)=\mu_{\text {res }}\left(B-B_{\text {res }}^{\left(m_{z}\right)}\right)$ of $\left|\phi_{\text {res }}^{\left(m_{z}\right)}\right\rangle$ is determined by the strength of the magnetic field. The difference between $B_{\text {res }}^{\left(m_{z}\right)}$ with $m_{z}=0, \pm 1$ depends on the atomic magnetic dipole. For the atoms with small magnetic dipole, e.g., ${ }^{6} \mathrm{Li}$, the values of $B_{\text {res }}^{(0)}$ and $B_{\text {res }}^{( \pm 1)}$ are close with each other [53], while for the the atoms with large magnetic dipole, e.g. ${ }^{40} \mathrm{~K}$, the difference between $B_{\text {res }}^{(0)}$ and $B_{\text {res }}^{( \pm 1)}$ is quite large [5].

The p-wave scattering amplitude in the open channel can be defined with the standard scattering theory [66]. To this end we firstly introduce the two-component stationary scattering state

$$
\left|\Phi_{\vec{k}}^{(+)}\right\rangle \equiv\left(\begin{array}{c}
\left|\phi_{\vec{k}}^{(\mathrm{op}+)}\right\rangle \\
\left|\phi_{\vec{k}}^{(\mathrm{cl}+)}\right\rangle
\end{array}\right)=\Omega_{+}\left(\begin{array}{c}
|\vec{k}\rangle \\
0
\end{array}\right)
$$

where $|\vec{k}\rangle$ is the eigenstate of the atomic relative momentum with eigen value $\hbar \vec{k}$, and $\Omega_{+}$is the Møller operator defined as

$$
\Omega_{+}=\lim _{\tau \rightarrow \infty} e^{-i H \tau / \hbar} e^{i \hat{T} \tau / \hbar} .
$$

In the large interatomic distance limit $r \rightarrow \infty$, the asymptotic behavior of the state $\left|\Phi_{\vec{k}}^{(+)}\right\rangle$can be expressed as

$$
\left\langle\vec{r} \mid \Phi_{\vec{k}}^{(+)}\right\rangle=\frac{1}{(2 \pi \hbar)^{\frac{3}{2}}}\left(\begin{array}{c}
e^{i \vec{k} \cdot \vec{r}}+f(\hat{r}, \vec{k}) \frac{e^{i k r}}{r} \\
0
\end{array}\right)
$$

with $\hat{r}=\vec{r} / r$ and $f(\hat{r}, \vec{k})$ the scattering amplitude which can be further expanded in terms of different partial waves:

$$
f(\hat{r}, \vec{k})=4 \pi \sum_{l m_{z}} f_{l m_{z}}(k) Y_{l}^{m_{z}}(\hat{r}) Y_{l}^{m_{z}}(\hat{k})^{*} .
$$

Here $Y_{l}^{m_{z}}(\hat{r})$ is the spherical harmonic function. For a scattering potential with $\mathrm{SO}(3)$ symmetry, the partial wave scattering amplitude only depends on the quantum number of the angular momentum $l$. In our case, the $\mathrm{SO}(3)$ symmetry is broken by the interaction between the atomic magnetic dipole and the magnetic field. Then we have a $m_{z}$-dependent scattering amplitude $f_{l m_{z}}(k)$.

In the case of low-energy scattering between two spin polarized fermonic atoms, one can neglect all the high partial wave scattering amplitudes $f_{l m_{z}}(k)$ with $l \geqslant 2$, and only consider the p-wave amplitudes $f_{1 m_{z}}(k)$, which can be further expressed in terms of the p-wave phase shifts $\delta_{1 m_{z}}(k)$ :

$$
f_{1 m_{z}}(k)=-\frac{1}{i k-k \cot \delta_{1 m_{z}}(k)} .
$$

During the scattering process, the bare bound state $\left|\phi_{\mathrm{res}}^{\left(m_{z}\right)}\right\rangle$ is coupled with the p-wave background scattering states in the open channel and significantly change the p-wave scattering amplitude $f_{1 m_{z}}(k)$. This effect can be directly treated via the Feshbach resonance theory, e.g., the methods in Ref. [67] and Ref. [69].

After a straightforward calculation in Appendix A, we find that the final phase shift $\delta_{1 m_{z}}(k)$ is the sum of the background phase shift $\delta_{1}^{(\mathrm{bg})}(k)$ for the background potential $V^{(\mathrm{bg})}(r)$ and a correction $\Delta_{1 m_{z}}(k)$ given by the closed channel:

$$
\delta_{1}(k)=\delta_{1}^{(\mathrm{bg})}(k)+\Delta_{1 m_{z}}(k) .
$$

Here $\Delta_{1 m_{z}}(k)$ satisfies

$$
-k \cot \Delta_{1 m_{z}}(k)=\frac{k}{\pi} \frac{\hbar^{2} k^{2} / m-E_{m_{z}}^{(\mathrm{cl})}(B)-g_{m_{z}}\left(k^{2}\right)}{\left|\left\langle\phi_{\mathrm{res}}^{\left(m_{z}\right)}|W| \psi_{k 1 m_{z}}^{(\mathrm{bg}+)}\right\rangle\right|^{2}}
$$

with $\left|\psi_{k 1 m_{z}}^{(\mathrm{bg}+)}\right\rangle$ and $g_{m_{z}}\left(k^{2}\right)$ given by

$$
\begin{aligned}
\left|\phi_{\vec{k}}^{(\mathrm{bg}+)}\right\rangle & =\left(\frac{2}{m \hbar k}\right)^{\frac{1}{2}} \sum_{l, m_{z}}\left|\psi_{k 1 m_{z}}^{(\mathrm{bg}+)}\right\rangle Y_{l}^{m_{z}}(\hat{k})^{*}, \\
g_{m_{z}}\left(k^{2}\right) & =\operatorname{Re}\left\langle\phi_{\mathrm{res}}^{\left(m_{z}\right)}\left|W G_{+}^{(\mathrm{bg})}\left(k^{2}\right) W\right| \phi_{\mathrm{res}}^{\left(m_{z}\right)}\right\rangle .
\end{aligned}
$$

In the above we have used the background Green's function $G_{+}^{(\mathrm{bg})}\left(k^{2}\right)$

$$
G_{+}^{(\mathrm{bg})}\left(k^{2}\right)=\frac{1}{\hbar^{2} k^{2} / m+i 0^{+}-\hat{T}-V^{(\mathrm{bg})}} .
$$

and the background scattering state $\left|\phi_{\vec{k}}^{(\mathrm{bg}+)}\right\rangle$ defined as

$$
\left|\phi_{\vec{k}}^{(\mathrm{bg}+)}\right\rangle=|\vec{k}\rangle+G_{+}^{(\mathrm{bg})}\left(k^{2}\right) V^{(\mathrm{bg})}|\vec{k}\rangle .
$$


In the following subsections, we evaluate the lowenergy expression of the scattering amplitude $f_{1 m_{z}}(k)$ by expanding the term $-k \cot \delta_{1 m_{z}}(k)$ in Eq. (9) in the limit $k \rightarrow 0$. As shown in Eq. (10), the phase shift $\delta_{1 m_{z}}(k)$ is the sum of $\delta_{1}^{(\mathrm{bg})}(k)$ and $\Delta_{1 m_{z}}(k)$. The low energy behavior of background phase shift $\delta_{1}^{(\mathrm{bg})}(k)$ is already known to be [1, 2]

$$
-k \cot \delta_{1}^{(\mathrm{bg})}(k)=\frac{1}{\mathcal{V}^{(\mathrm{bg})}} \frac{1}{k^{2}}+\frac{1}{\mathcal{S}^{(\mathrm{bg})}} \frac{1}{k}+\frac{1}{\mathcal{R}^{(\mathrm{bg})}} .
$$

Therefore, if we can further obtain the low-energy expansion of term $-k \cot \Delta_{1 m_{z}}(k)$, then the expressions of $-k \cot \delta_{1 m_{z}}(k)$ and $f_{1 m_{z}}(k)$ can be calculated straightforwardly.

\section{B. The low-energy expansion of $-k \cot \Delta_{1 m_{z}}(k)$}

In this subsection we investigate the expression of $-k \cot \Delta_{1 m_{z}}(k)$ in the limit $k \rightarrow 0$. To this end, we need to expand both the numerator and the denominator of (11) in the low-energy limit.

In this paper we assume the background scattering volume in the open channel is finite. It can be proved that (Appendix C), in this case the function $g_{m_{z}}\left(k^{2}\right)$ can be expanded as

$$
g_{m_{z}}\left(k^{2}\right)=g_{m_{z}}^{(0)}+g_{m_{z}}^{(2)} k^{2}+O\left(k^{3}\right)
$$

with $g_{m_{z}}^{(2)} \leq 0$.

On the other hand, due to the long-range nature of the van der Waals potential, the partial wave scattering state $\left|\psi_{k 1 m_{z}}^{(\mathrm{bg}+)}\right\rangle$ is not an analytical function of the incident momentum $k$ in the neighborhood of $k=0$ [66]. To investigate the low-energy behavior of $\left|\psi_{k 1 m_{z}}^{(\mathrm{bg}+)}\right\rangle$ and then the denominator of (11), we separate the non-analytical part of $\left|\psi_{k 1 m_{z}}^{(\mathrm{bg}+)}\right\rangle$ by introducing the background Jost function $\mathscr{J}(k)[66]$ defined as

$$
\left\langle\vec{r} \mid \psi_{k 1 m_{z}}^{(\mathrm{bg}+)}\right\rangle=i^{l} \frac{1}{\hbar \mathscr{J}(k)}\left(\frac{m}{\pi k}\right)^{\frac{1}{2}} \frac{1}{r} Y_{1}^{m_{z}}(\hat{r}) \tilde{F}_{k 1 m_{z}}^{(\mathrm{bg})}(r) .
$$

Here $\tilde{F}_{k 1 m_{z}}^{(\mathrm{bg})}(r)$ is the canonical solution of the radial equation

$$
\left(-\frac{d^{2}}{d r^{2}}+V^{(\mathrm{bg})}(r)+\frac{2}{r^{2}}\right) \tilde{F}_{k 1 m_{z}}^{(\mathrm{bg})}(r)=k^{2} \tilde{F}_{k 1 m_{z}}^{(\mathrm{bg})}(r)
$$

with boundary condition

$$
\tilde{F}_{k 1 m_{z}}^{(\mathrm{bg})}(r \rightarrow 0) \rightarrow \hat{\jmath}_{1}(k r)
$$

where

$$
\hat{\jmath}_{1}(x)=\frac{\sin x}{x}-\cos x
$$

is the first-order regular Riccati-Bessel function [66]. According to the standard scattering theory [66], $\tilde{F}_{k 1 m_{z}}^{(\mathrm{bg})}(r)$ is an analytical function of $k$, and can be expanded as a Talyor series of $k$ :

$$
\tilde{F}_{k 1 m_{z}}^{(\mathrm{bg})}(r)=\left.\frac{1}{(2 n) !} \sum_{n=1}^{\infty} \frac{d^{2 n}}{d k^{2 n}} \tilde{F}_{k 1 m_{z}}^{(\mathrm{bg})}(r)\right|_{k=0} k^{2 n}
$$

It is pointed out that, all the odd order terms of the above Taylor series are exactly zero [66]. Thus the nonanalytical part of $\left|\psi_{k 1 m_{z}}^{(\mathrm{bg}+)}\right\rangle$ is included in the term with the Jost function $\mathscr{J}(k)$.

Substituting Eqs. (22), (18) and (17) into Eq. (11), we find that in the low-energy limit the factor $-k \cot \Delta_{1 m_{z}}(k)$ takes the form

$$
-k \cot \Delta_{1 m_{z}}(k)=\frac{1}{\mathcal{V}^{(\Delta)}\left(B ; k ; m_{z}\right)} \frac{1}{k^{2}}+\frac{1}{\mathcal{R}^{(\Delta)}\left(B ; k ; m_{z}\right)} .
$$

Here we have the $k$-dependent parameters:

$$
\begin{aligned}
\mathcal{V}^{(\Delta)}\left(B ; k ; m_{z}\right)= & -|\mathscr{J}(k)|^{-2} \frac{\pi w_{m_{z}}}{\mu_{\mathrm{res}}} \frac{1}{B-B_{0}} ; \\
\mathcal{R}^{(\Delta)}\left(B ; k ; m_{z}\right)= & |\mathscr{J}(k)|^{-2} \pi w_{m_{z}} \times \\
& {\left[\left(\frac{\hbar^{2}}{m}-g_{m_{z}}^{(2)}\right)-\frac{w_{m_{z}}^{\prime}}{w_{m_{z}}} \mu_{\mathrm{res}}\left(B-B_{0}\right)\right]^{-1} . }
\end{aligned}
$$

with the parameters $B_{0}$ and $w_{m_{z}}$ defined by

$$
\begin{aligned}
B_{0} & =B_{\mathrm{res}}-g_{m_{z}}^{(0)} / \mu_{\mathrm{res}} ; \\
w_{m_{z}} & =\frac{1}{6} \frac{d^{3}}{d k^{3}}\left[\left|\left\langle\phi_{\mathrm{res}}^{\left(m_{z}\right)}|W| \psi_{k 1 m_{z}}^{(\mathrm{bg}+)}\right\rangle\right|^{2} \times|\mathscr{J}(k)|^{2}\right]_{k=0} \\
w_{m_{z}}^{\prime} & =\frac{1}{120} \frac{d^{5}}{d k^{5}}\left[\left|\left\langle\phi_{\mathrm{res}}^{\left(m_{z}\right)}|W| \psi_{k 1 m_{z}}^{(\mathrm{bg}+)}\right\rangle\right|^{2} \times|\mathscr{J}(k)|^{2}\right]_{k=0} .
\end{aligned}
$$

\section{The low-energy p-wave scattering amplitude}

In the above subsection we get the expansion (23) of the factor $-k \cot \Delta_{1 m_{z}}(k)$. Substituting Eq. (23), (16) and (10) into (9), we finally get the low-energy behavior of the p-wave scattering amplitude $f_{1 m_{z}}(k)$ in presence of a PMFR:

$$
\begin{aligned}
& f_{1 m_{z}}(k) \\
= & -\frac{1}{i k+\frac{1}{\mathcal{V}^{\text {eff }}\left(k ; B ; m_{z}\right) k^{2}}+\frac{1}{\mathcal{S}^{\text {eff }}\left(k ; B ; m_{z}\right) k}+\frac{1}{\mathcal{R}^{\text {eff }}\left(k ; B ; m_{z}\right)}}
\end{aligned}
$$


where the $k$-dependent scattering parameters are given by

$$
\begin{aligned}
\mathcal{V}^{\mathrm{eff}} & =\mathcal{V}^{(\mathrm{bg})}\left(1-\frac{b_{m_{z}}}{B-B_{0}}|\mathscr{J}(k)|^{-2}\right) \\
\mathcal{S}^{\mathrm{eff}} & =\frac{\mathcal{S}^{(\mathrm{bg})}}{\mathcal{V}^{(\mathrm{bg}) 2}} \mathcal{V}^{\mathrm{eff} 2} \\
\frac{1}{\mathcal{R}^{\mathrm{eff}}} & =\frac{1}{\mathcal{R}^{(\Delta)}}\left(1-2 x+x^{2}\right)+\frac{1}{\mathcal{R}^{(\mathrm{bg})}} x^{2}+\frac{\mathcal{V}^{(\mathrm{bg})}}{\mathcal{S}^{(\mathrm{bg}) 2}}\left(x^{2}-x^{3}\right)
\end{aligned}
$$

Here the parameters $b_{m_{z}}$ and $x$ are defined as

$$
b_{m_{z}}=\frac{\pi w_{m_{z}}}{\mathcal{V}^{(\mathrm{bg})} \mu_{\mathrm{res}}} ; x=\frac{\mathcal{V}^{(\mathrm{bg})}}{\mathcal{V}^{\mathrm{eff}}} .
$$

So far we have obtained the low-energy expression of the p-wave scattering amplitude $f_{1 m_{z}}(k)$ in the case of PMFR. With the help of the scattering theory, we obtain the general expressions (28,29) for the scattering amplitude $f_{1 m_{z}}(k)$ as well as the scattering parameters $\left(\mathcal{V}^{\text {eff }}, \mathcal{S}^{\text {eff }}, \mathcal{R}^{\text {eff }}\right)$, which are formulated in terms of the background scattering parameters $\left(\mathcal{V}^{(\mathrm{bg})}, \mathcal{S}^{(\mathrm{bg})}, \mathcal{R}^{(\mathrm{bg})}\right)$ and the magnetic field. It is pointed out that, although due to the long-range nature of the van der Waals potential we can not express the denominator of $f_{1 m_{z}}(k)$ as an Laurent series with $k$-independent coefficients, we successfully include all the $k$-dependence of the parameters $\left(\mathcal{V}^{\text {eff }}, \mathcal{S}^{\text {eff }}, \mathcal{R}^{\text {eff }}\right)$ into the Jost function $\mathscr{J}(k)$.

Eq. (29) clearly show the effect of PMFA that the scattering volume $V^{\text {eff }}$ diverges under the magnetic field $B=B_{0}$. In the realistic cold atom gases, for the creation of an observable effects with $p$-wave interaction, the scattering volume $\left|V^{\text {eff }}\right|$ should be large enough. Particularly, $\left|V^{\text {eff }}\right|^{1 / 3}$ should be much larger than the van der Waals length so that in the BCS region, the transition temperature $T_{c} \sim\left(E_{F} / k_{B}\right) \exp \left[-\pi /\left(2 k_{B}^{3} V^{\text {eff }}\right)\right][71]$ of superfluid is realizable and in the $\mathrm{BEC}$ region the binding energy of the $p$-wave Feshbach molecule be roubst with respect to the detail of the atom-atom interaction potential.

In the end of this section, we consider the dependence of the effective range $R^{\text {eff }}$ on the magnetic field $B$. According to Eq. (29), $R^{\text {eff }}$ depends on $B$ through the ratio $x$ between $V^{(\mathrm{bg})}$ and $V^{\mathrm{eff}}$, and the quantatity $R^{(\Delta)}\left(B ; k ; m_{z}\right)$. In the cold gases of ${ }^{6} \mathrm{Li}$ and ${ }^{40} \mathrm{~K}$, the background scattering volumes $V^{(\mathrm{bg})}$ are of the order of $\left(10^{5}-10^{6}\right) a_{0}^{3}$. According to our above discussion, they are too small for the creation of $p$-wave superfluids 71]. Therefore in these systems the strong enough $p$-wave interactions can only be obtained in the resonance region with $\left|V^{\text {eff }}\right|>>\left|V^{(\mathrm{bg})}\right|$ or $x<<1$, which implies $R^{\mathrm{eff}} \approx R^{(\Delta)}\left(B ; k ; m_{z}\right)$. On the other hand, according to Eq. (25), the dependence of $R^{(\Delta)}\left(B ; k ; m_{z}\right)$ on $B$ is significant when the magnetic field is far away enough from from the resonant point $B_{0}$ so that the factor $\left|w_{m_{z}}^{\prime} \mu_{\text {res }}\left(B-B_{0}\right) / w_{m_{z}}\right|$ is comparable or larger than $\left|\hbar^{2} / m-g_{m_{z}}^{(2)}\right|$. The values of $w_{m_{z}}^{\prime}$ and $w_{m_{z}}$ are not available for ${ }^{6} \mathrm{Li}$ and ${ }^{40} \mathrm{~K}$. Nevertheless, the binding energies of the $p$-wave Feshbach molecules are measured to be linear functions of the magentic field [7, 11] in the region with large enough $p$-wave scattering volumes $\left(V^{\text {eff }} \gtrsim 10^{7} a_{0}^{3}\right)$. This observation shows that in these regions the term $\left|w_{m_{z}}^{\prime} \mu_{\text {res }}\left(B-B_{0}\right) / w_{m_{z}}\right|$ is negligible and the effective range $R^{\text {eff }}$ can be approximated as a $B$-independent constant $R^{(\Delta)}\left(0 ; k ; m_{z}\right)$.

\section{THE APPLICABILITY OF THE EFFECTIVE-RANGE THEORY}

In the above section, we have obtained the expression (28) of the p-wave scattering amplitude $f_{1 m_{z}}(k)$ in the region near the point of PMFR. It is apparent that, this expression is different from the one (1) given by the effective-range theory in the following two senses:

1. In the standard effective-range theory, the scattering volume $\mathcal{V}$ and effective-range $\mathcal{R}$ are independent on the incident momentum $k$. Nevertheless, in the expression (28) the scattering parameters $\left(\mathcal{V}^{\mathrm{eff}}, \mathcal{S}^{\mathrm{eff}}, \mathcal{R}^{\mathrm{eff}}\right)$ depend on $k$ through the Jost function $\mathscr{J}(k)$.

2. The term $1 /\left[\mathcal{S}^{\mathrm{eff}}\left(k ; B ; m_{z}\right) k\right]$ cannot be included in the effective-range theory.

It is apparent that, if under some condition the scattering amplitude (28) can be approximated as

$$
f_{1 m_{z}}(k) \approx-\frac{1}{i k+\frac{1}{\mathcal{V}^{\text {eff }}\left(0 ; B ; m_{z}\right) k^{2}}+\frac{1}{\mathcal{R}^{\text {eff }}\left(0 ; B ; m_{z}\right)}},
$$

i.e., both the $k$-dependence of $|\mathscr{J}(k)|^{2}$ and the term $1 /\left[\mathcal{S}^{\mathrm{eff}}\left(k ; B ; m_{z}\right) k\right]$ can be neglected, the behavior of the system would be approximately described by the effective-range theory. In this section, we investigate the conditions for the approximation (32) or (??), or the applicability of effective-range theory. We will consider the importance of the term $1 /\left[\mathcal{S}^{\text {eff }}\left(k ; B ; m_{z}\right) k\right]$ and the $k$ dependence of the Jost function respectively.

\section{A. The $k$-dependence of the scattering parameters}

In this subsection we search the sufficient condition for the ignorance of the $k$-dependence of the scattering parameters $\left(\mathcal{V}^{\text {eff }}, \mathcal{S}^{\text {eff }}, \mathcal{R}^{\text {eff }}\right)$. As we have discussed above, the $k$-dependence of the scattering parameters comes from the mode square of the Jost function $\mathscr{J}(k)$. In the ultracold gases of the fermionic atoms, the maximum value of the relative momentum of two atoms is on the order of the Fermi momentum $k_{F}$. Therefore, the importance of the $k$-dependence of the parameters parameters $\left(\mathcal{V}^{\mathrm{eff}}, \mathcal{S}^{\mathrm{eff}}, \mathcal{R}^{\mathrm{eff}}\right)$ can be described by the factor

$$
r_{1}=\frac{\left|\mathscr{J}\left(k_{F}\right)\right|^{-2}-|\mathscr{J}(0)|^{-2}}{|\mathscr{J}(0)|^{-2}} .
$$




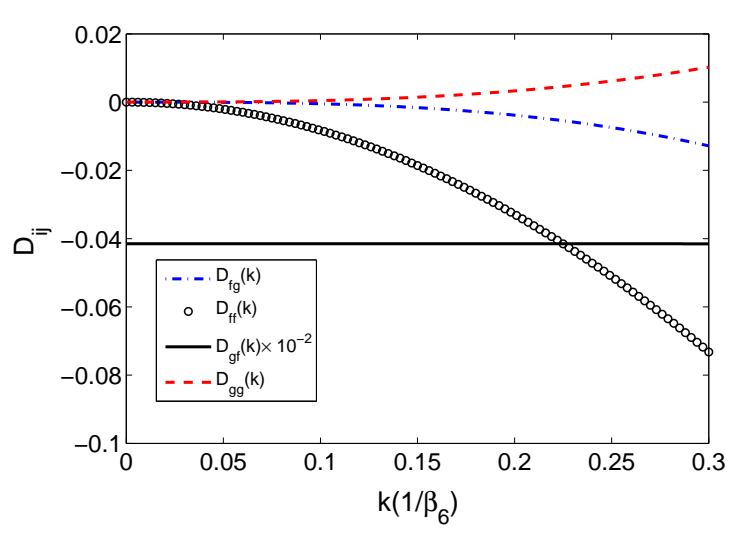

FIG. 2: (color online) The functions $D_{f g}(k)$ (blue dash-dotted line), $D_{f f}(k)$ (open circle), $D_{g f}(k)$ (black solid line) and $D_{g g}(k)$ (red dashed line) in Eq. (34).

Obviously, when $r_{1}<<1$, we can replace $|\mathscr{J}(k)|^{-2}$ with $|\mathscr{J}(0)|^{-2}$ and neglect the $k$ - dependence of the parameters $\left(\mathcal{V}^{\text {eff }}, \mathcal{S}^{\text {eff }}, \mathcal{R}^{\text {eff }}\right)$.

To investigate the behavior of the the ratio $r_{1}$, we first calculate the Jost function $\mathscr{J}(k)$. By means of the quantum defect theory 74], we can obtain the expression of $|\mathscr{J}(k)|^{-2}$ (see Appendix D):

$$
\begin{aligned}
& |\mathscr{J}(k)|^{-2}=\alpha^{-2} \beta_{6}^{3} \frac{\pi}{2} \times \\
& {\left[\left(D_{f f}(k)-K_{l=1}^{0} D_{g f}(k)\right)^{2}+\left(D_{f g}(k)-K_{l=1}^{0} D_{g g}(k)\right)^{2}\right]}
\end{aligned}
$$

where $\alpha$ is a $k$-independent coefficient and $D_{i j}(k)=$ $\left(k \beta_{6}\right)^{3 / 2} Z_{i j}(k)(i, j=f, g)$ with $Z_{i j}(k)$ defined in 68]. In Fig. 2 we plot the functions $D_{i j}(k)$ in the low-energy case.

The parameter $K_{l=1}^{0}$ is denoted as $K_{l=1}^{0}$ is related to the background scattering parameters [2]. Expanding the p-wave phase shift in Eq.(7) of Ref. [2], we can express $\left(\mathcal{V}^{(\mathrm{bg})}, \mathcal{S}^{(\mathrm{bg})}\right)$ in terms of $K_{l=1}^{0}$ :

$$
\begin{aligned}
& \mathcal{V}^{(\mathrm{bg})}=-\frac{\left(1+K_{l=1}^{0}\right) \pi}{18 K_{l=1}^{0} \Gamma[3 / 4]^{2}} \beta_{6}^{3} ; \\
& \mathcal{S}^{(\mathrm{bg})}=-\frac{35\left(1+K_{l=1}^{0}\right)^{2} \pi}{324\left(K_{l=1}^{0}\right)^{2} \Gamma[3 / 4]^{4}} \beta_{6}^{2} .
\end{aligned}
$$

The above expression shows that, when $K_{l=1}^{0} \gtrsim$ $\pi /\left(18 \Gamma[3 / 4]^{2}\right) \sim 0.1$, we have $\mathcal{V}^{(\mathrm{bg})} \sim \beta_{6}^{3}$ and the background scattering potential is far away from the shape resonance; when $K_{l=1}^{0}$ is much smaller than 0.1, the background potential is in the shape resonance region which gives $\mathcal{V}^{(\mathrm{bg})}>>\beta_{6}^{3}$.

Now we consider the features of the ratio $r_{1}$, which is determined by the parameter $K_{l=1}^{0}$. Fig. 2 shows that in the low energy case with $k \beta_{6}<<1$, the function $D_{g f}(k)$ is almost a $k$-independent constant and much larger than the other three D-functions. Therefore, if the parameter

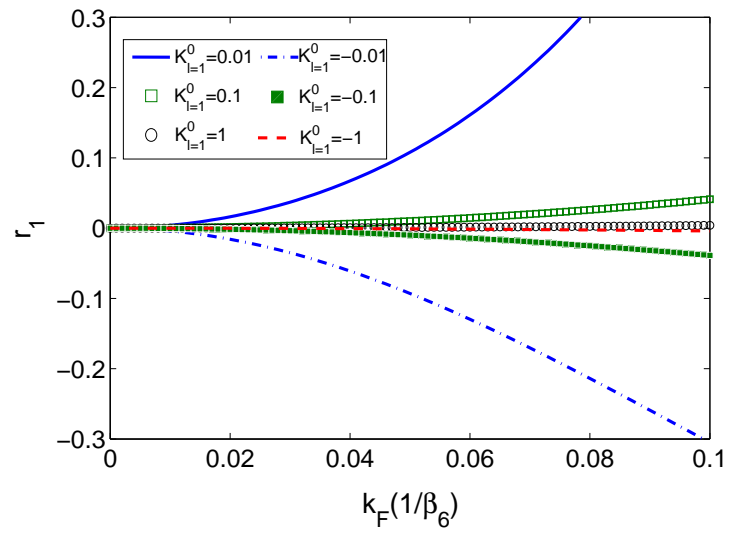

FIG. 3: (color online) Ratio $r_{1}$ defined in Eq. (33) as a function of the Fermi momentum $k_{F}$ with $K_{l=1}^{0}=0.01\left(\mathcal{V}^{(\mathrm{bg})}=\right.$ $-11.79 \beta_{6}^{3}$, blue solid line $), K_{l=1}^{0}=0.1\left(\mathcal{V}^{(\mathrm{bg})}=-1.27 \beta_{6}^{3}\right.$, green empty square), $K_{l=1}^{0}=1\left(\mathcal{V}^{(\mathrm{bg})}=-0.23 \beta_{6}^{3}\right.$, black empty circle), $K_{l=1}^{0}=-0.01\left(\mathcal{V}^{(\mathrm{bg})}=11.51 \beta_{6}^{3}\right.$, blue dashdotted line $), K_{l=1}^{0}=-0.1\left(\mathcal{V}^{(\mathrm{bg})}=10.4 \beta_{6}^{3}\right.$, green filled square), $K_{l=1}^{0}=-1\left(\mathcal{V}^{(\mathrm{bg})}=0\right.$, red dashed line $)$.

$K_{l=1}^{0}$ is large or the background scattering potential in the open channel is far away from the shape resonance, then according to Eq. (34), the Jost function $|\mathscr{J}(k)|$ is dominated by the term with $K_{l=1}^{0} D_{g f}(k)$. In this case the variation of $|\mathscr{J}(k)|^{-2}$ with respect to $k$ is negligi]$^{-1}$ ble and we have $r_{1}<<1$. On the other hand, if $K_{l=1}^{0}$ is close to zero and the background scattering potential (34)is close to the shape resonance, then $|\mathscr{J}(k)|$ becomes a rapid changing function of $k$ and the ratio $r_{1}$ would be significant.

The above argument is quantitatively verified by Fig. 2 , where the ratio $r_{1}$ is plotted as functions of $k$ with respect different values of $K_{l=1}^{0}$ or $\mathcal{V}^{\text {(bg) }}$. It is clearly shown that, if the fermonic momentum $k_{F} \lesssim 0.1 \beta_{6}^{-1}$, then the ratio $r_{1}$ and the $k$-dependence of the scattering parameters can be neglected when the background potential is far enough from the shape resonance so that $\mathcal{V}^{(\mathrm{bg})} \lesssim \beta_{6}^{3}$. If $k_{F} \lesssim 0.01 \beta_{6}^{-1}$, this restriction can be further relaxed to $\mathcal{V}^{(\mathrm{bg})} \lesssim 10 \beta_{6}^{3}$.

\section{B. The importance of the term $1 /\left[\mathcal{S}^{\text {eff }}\left(k ; B ; m_{z}\right) k\right]$}

Now we discuss the importance of the term $1 /\left[\mathcal{S}^{\mathrm{eff}}\left(k ; B ; m_{z}\right) k\right]$. Since the purpose of this paper is to obtain the sufficient condition for the effective-range theory, or the approximation (32), for simplicity, in this subsection we assume the condition $r_{1}<<1$ obtained in the above section is already met, and the $k$-dependence of the coefficients $\left(\mathcal{V}^{\text {eff }}, \mathcal{S}^{\text {eff }}, \mathcal{R}^{\text {eff }}\right)$ can be neglected.

In the BEC side of the PMFR where $B<B_{0}$, since $g_{m_{z}}^{(2)}<0$, the parameters $\mathcal{R}^{\text {eff }}$ and $\mathcal{V}^{\text {eff }}$ have the same sign. In that case, if the absolute value of $1 /\left(\mathcal{S}^{\text {eff }} k\right)$ is much smaller than the one of $1 /\left(\mathcal{V}^{\text {eff }} k^{2}\right)$, then it would 
be also much smaller than $1 /\left(\mathcal{V}^{\text {eff }} k^{2}\right)+1 / \mathcal{R}^{\text {eff }}$, and can be neglected. It is obvious that, in the limit $k \rightarrow 0$, the term $1 /\left(\mathcal{S}^{\text {eff }} k\right)$ would be much smaller than $1 /\left(\mathcal{V}^{\text {eff }} k^{2}\right)$. Therefore the importance of the term $1 /\left(\mathcal{S}^{\text {eff }} k\right)$ is actually determined by the ratio $r_{2}$ between the two terms for the upper limit of the relative momentum $k_{F}$ :

$$
r_{2}=\left|\frac{1 /\left[\mathcal{S}^{\mathrm{eff}}\left(0 ; B ; m_{z}\right) k_{F}\right]}{1 /\left[\mathcal{V}^{\text {eff }}\left(0 ; B ; m_{z}\right) k_{F}^{2}\right]}\right| .
$$

When $r_{2}$ is much smaller than unity, we can neglect the term $1 /\left(\mathcal{S}^{\text {eff }} k\right)$. If $r_{2}$ is comparable or larger than unity, the term $1 /\left(\mathcal{S}^{\text {eff }} k\right)$ would be necessary for the theory.

The straightforward calculation with Eqs. (29), (30), (35) and (36) yields

$$
r_{2}=\frac{\pi}{35} \frac{\beta_{6}^{3}}{\left|\mathcal{V}^{\text {eff }}\left(k_{F} ; B ; m_{z}\right)\right|}\left(\beta_{6} k_{F}\right) .
$$

In the practical cold atom systems we have $\beta_{6} k_{F}<<1$. Therefore, in the resonance region with $\mathcal{V}^{\text {eff }} \gtrsim \beta_{6}^{3}$, the ratio $r_{2}$ in Eq. (38) is much smaller than unity, and then the term $1 /\left(\mathcal{S}^{\text {eff }} k\right)$, is negligible.

In the BEC side of the resonance with $B>B_{0}$, the parameters $\mathcal{V}^{\text {eff }}$ and $\mathcal{R}^{\text {eff }}$ have different signs. In that case, there is a speical momentum

$$
k_{*}=\sqrt{-\frac{\mathcal{R}^{\mathrm{eff}}\left(0 ; B ; m_{z}\right)}{\mathcal{V}^{\mathrm{eff}}\left(0 ; B ; m_{z}\right)}}
$$

which makes the terms $1 /\left[\mathcal{V}^{\text {eff }} k_{*}^{2}\right]$ and $1 /\left[\mathcal{R}^{\text {eff }}\right]$ cancel with each other or

$$
\frac{1}{\mathcal{V}^{\mathrm{eff}}\left(0 ; B ; m_{z}\right) k_{*}^{2}}+\frac{1}{\mathcal{R}^{\mathrm{eff}}\left(0 ; B ; m_{z}\right)}=0 .
$$

Therefore, if the atomic relative momentum $k$ is far away from $k_{*}$, the absolute value of $1 /\left(\mathcal{R}^{\text {eff }} k^{2}\right)$ would be quite different with the one of $1 / \mathcal{V}^{\text {eff }}$. In that case we can still neglect the term $1 /\left(\mathcal{S}^{\text {eff }} k\right)$ under the condition $r_{2}<<1$ or $\left|1 /\left(\mathcal{S}^{\text {eff }} k\right)\right|<<\left|1 /\left(\mathcal{R}^{\text {eff }} k^{2}\right)\right|$.

If the atomic relative momentum $k$ is in the neighborhood of $k_{*}$ and the terms $1 /\left(\mathcal{R}^{\mathrm{eff}} k_{*}^{2}\right)$ is canceled with $1 / \mathcal{V}^{\text {eff }}$, the scattering amplitude (28) can be expressed as

$$
f_{1 m_{z}}(k)=-\frac{1}{i k_{*}+\frac{1}{\mathcal{S}^{\mathrm{eff}}\left(0 ; B ; m_{z}\right) k_{*}}} .
$$

In that case, if the absolute value of $1 /\left[\mathcal{S}^{\text {eff }}\left(0 ; B ; m_{z}\right) k_{*}\right]$ is much smaller than $k_{*}$, we an also neglect the term with $\mathcal{S}^{\text {eff }}$, even in the neighborhood of $k_{*}$. We define a parameter $r_{3}$ as

$$
r_{3}=\frac{1}{\left|\mathcal{S}^{\text {eff }}\left(0 ; B ; m_{z}\right) k_{*}^{2}\right|} .
$$

Then the term with $\mathcal{S}^{\text {eff }}$ can be neglected when $r_{2,3}<<1$. A further calculation with Eqs. (39), (29), (30), (35) and (36) implies that

$$
r_{3}=\frac{\pi \beta_{6}^{4}}{35\left|\mathcal{V}^{\mathrm{eff}}\left(0 ; B ; m_{z}\right) \mathcal{R}^{\mathrm{eff}}\left(0 ; B ; m_{z}\right)\right|}
$$

As shown above, the condition $r_{3}<<1$ is obtained for the momentum region $k \sim k_{*}$. Since the realistic momentum of the atomic relative motion takes the value between zero and $k_{F}$, in the cases with $k_{F}<k_{*}$, we can disregard the restriction of the ratio $r_{3}$, and use effectiverange theory under the condition $r_{1,2}<<1$ in both the $\mathrm{BEC}$ and the BCS sides of the resonance.

\section{Summary}

In summary, the general sufficient conditions for the effective-range theory in the BCS side of the resonance can be summarized as

$$
r_{1}, r_{2}, r_{3}<<1
$$

while the ones for the BEC side are

$$
r_{1}, r_{2}<<1
$$

From the definition of the ratios $r_{1}, r_{2}$ and $r_{3}$, we notice that in the realistatic cold gases of Fermi atoms, the crucial factors for the usage of effective range theory is the background $p$-wave scattering volume $\mathcal{V}^{\text {(bg) }}$ and the $B$-dependence of the factor $\mathcal{R}^{(\Delta)}\left(0 ; B ; m_{z}\right)$. If the the background $p$-wave scattering is far away from the shape resonance so that $\mathcal{V}^{(\mathrm{bg})} \sim \beta_{6}^{3} \sim\left(100 a_{0}\right)^{3}$ then according to our previous discussions and Eqs. (38), the conditions $r_{1}, r_{2}<<1$ can be satisfied in the region $\mathcal{V}^{\text {eff }} \gtrsim$ $10 \beta_{6}^{3} \sim 10^{7} a_{0}^{3}$ where the $p$-wave interaction is strong enough for the creation of $p$-wave superfluids. In that region we also have $\mathcal{R}^{\text {eff }}\left(0 ; B ; m_{z}\right)=\mathcal{R}^{(\Delta)}\left(0 ; B ; m_{z}\right)$. If $\mathcal{R}^{(\Delta)}\left(0 ; B ; m_{z}\right)$ can be further approximated as a $B$ independent constant which is of the order $\beta_{6}$, then the condition $r_{3}<<1$ can also be satisfied, and the effective range theory can be used a good approximation for the realistic scattering amplitude. In the following subsection we show that the PMFRs in the cold gases of ${ }^{40} \mathrm{~K}$ and ${ }^{6} \mathrm{Li}$ are just of this case.

\section{Discussion for the cold gases of ${ }^{40} \mathrm{~K}$ and ${ }^{6} \mathrm{Li}$}

In the above subsections we obtained the sufficient conditions (43), (44) of the effective-range theory for the p-wave scattering amplitudes of polarized fermonic atoms near a PMFR. In this subsection, with the help of the conditions, we perform a discussion on the usage of effective-range theory in the ultracold gases of ${ }^{40} \mathrm{~K}$ and ${ }^{6} \mathrm{Li}$.

For the ultracold gas with ${ }^{40} \mathrm{~K}$ atoms in the state $|9 / 2,-7 / 2\rangle$, we have $C_{6}=3897$ (a.u.) [75] and $\mathcal{V}^{(\mathrm{bg})}=$ $-10^{6} a_{0}^{3}$ [5]. These parameters leads $\beta_{6}=130 a_{0}$ and $K_{l=1}^{0}=-0.16$. If the Fermi temperature $T_{F}=1 \mu \mathrm{K}$, then we have $k_{F} \beta_{6}=0.06$. The straightforward calculation shows that $r_{1}=0.01$. Therefore $k$-dependence of the scattering parameters can be safely neglected. The p-wave Feshbach resonance for the states with $m_{l}= \pm 1$ 
occurs at $B_{0}=198.37 \mathrm{G}$ with width $\Delta B=25 \mathrm{G}$ and effective-range $\mathcal{R}^{\text {eff }}=47.2 a_{0}$. The resonance for the states with $m_{l}=0$ occurs at $B_{0}=198.85 \mathrm{G}$ with width $\Delta B=22 \mathrm{G}$ and effective-range $\mathcal{R}^{\mathrm{eff}}=46.2 a_{0}[5]$. According to these data we have $r_{3}<0.02$ when $k_{*}<k_{F}$. Then effect from the ratio $r_{3}$ is also negligible. Therefore the sufficient condition for the usage of effective-range simply becomes $r_{2}<<1$. Further calculation shows that $r_{2} \leq 0.013$ when $\left|\mathcal{V}^{\text {eff }}\right| \geq\left|\mathcal{V}^{(\mathrm{bg})}\right|$. Then the effectiverange approximation (32) is applicable for ${ }^{40} \mathrm{~K}$ atoms in the state $|9 / 2,-7 / 2\rangle$ in the whole region of PMFR with $\left|\mathcal{V}^{\text {eff }}\right| \geq\left|\mathcal{V}^{(\mathrm{bg})}\right|$. The condition for the effectiverange approximation is broken only in the small region $220.5 \mathrm{G}<B<221 \mathrm{G}\left(m_{l}=0\right)$ or $223 \mathrm{G}<B<223.7 \mathrm{G}$ $\left(m_{l}= \pm 1\right)$ where we have $\left|\mathcal{V}^{\text {eff }}\right| \leq 0.005 \beta_{6}^{3}$ or $r_{2} \geq 1$.

Now we consider the gas with ${ }^{6} \mathrm{Li}$ atoms in the ground hyperfine state $\left|F=1 ; m_{F}=1\right\rangle$. In that case we have $C_{6}=1393$ (a.u.) [70, 76] and $\mathcal{V}^{(\text {bg) }}=-\left(35.3 a_{0}\right)^{3}$. These parameters leads $\beta_{6}=62 a_{0}$ and $K_{l=1}^{0}=-0.38$. If the Fermi temperature $T_{F}=1 \mu \mathrm{K}$, we have $k_{F} \beta_{6}=0.01$ which implies $r_{1}=2 \times 10^{-4}$. Then similar as above, the effective range approximation (32) is also applicable for ${ }^{6} \mathrm{Li}$ atoms in the whole region of PMFR with $\left|\mathcal{V}^{\text {eff }}\right| \geq$ $\left|\mathcal{V}^{(\mathrm{bg})}\right|$

\section{CONCLUSION}

In this work we obtain the explicit expression of the p-wave scattering amplitude of two ultracold spin polarized fermonic atoms near the p-wave Feshbach resonance. We show that due to the long rang nature of the van der Waals potential, the scattering amplitude is explicitly described by Eq. (2) in the low-energy case. With the help of the quantum defect theory, we formulate all the scattering parameters $\left(\mathcal{V}^{\text {eff }}, \mathcal{S}^{\text {eff }}, \mathcal{R}^{\text {eff }}\right)$ in terms of the background parameters and the inter-channel coupling.

Based on this result, we discussed the applicability of the effective-range theory, or the Eq. (3) as an approximation of the exact scattering amplitude. We show that, in the BEC side of the resonance, the sufficient conditions of the effective-range theory can be quantitatively described as $r_{1}, r_{2}<<1$ while in the BCS side the conditions become $r_{1}, r_{2}, r_{3}<<1$, where $r_{1}, r_{2}, r_{3}$ are defined in Eqs. (38), (33), and (42). The applicability of the effective-range theory for the ultracold gases of ${ }^{40} \mathrm{~K}$ and ${ }^{6} \mathrm{Li}$ are examined with our results. The effective-range theory is shown to be a good approximation in both of the two cases in the resonance regime where the absolute value of the scattering volume is equal to or larger then the background one.

\section{Appendix A: The p-wave phase shift with PMFR}

In this appendix we post the derivation of the p-wave phase shift $\delta_{1 m_{z}}(k)$ in Eqs. (10) and (11). Our calculation is based on the method in Ref. 69]. We begin from the scattering state $\left|\Phi_{\vec{k}}^{(+)}\right\rangle$in Eq. (15). According to the scattering theory [66], the open channel component $\left|\phi_{\vec{k}}^{(\mathrm{op}+)}\right\rangle$ and closed channel component $\left|\phi_{\vec{k}}^{(\mathrm{cl})}\right\rangle$ of $\left|\Phi_{\vec{k}}^{(+)}\right\rangle$ satisfy the two channel Lippmman-Schwinger equation

$$
\begin{aligned}
\left|\phi_{\vec{k}}^{(\mathrm{op}+)}\right\rangle & =|\vec{k}\rangle+G_{0}^{(+)}\left(k^{2}\right) V^{(\mathrm{bg})}\left|\phi_{\vec{k}}^{(\mathrm{op}+)}\right\rangle+G_{0}^{(+)}\left(k^{2}\right) W\left|\phi_{\vec{k}}^{(\mathrm{cl}+)}\right\rangle \\
\left|\phi_{\vec{k}}^{(\mathrm{cl}+)}\right\rangle & =G_{0}^{(\mathrm{cl})}\left(k^{2}\right) V^{(\mathrm{cl})}\left|\phi_{\vec{k}}^{(\mathrm{cl}+)}\right\rangle+G_{0}^{(\mathrm{cl})}\left(k^{2}\right) W\left|\phi_{\vec{k}}^{(\mathrm{op}+)}\right\rangle
\end{aligned}
$$

with the free Green's functions

$$
\begin{aligned}
G_{0}^{(+)}\left(k^{2}\right) & =\frac{1}{\hbar^{2} k^{2} / m+i 0^{+}-\hat{T}} ; \\
G_{0}^{(\mathrm{cl})}\left(k^{2}\right) & =\frac{1}{\hbar^{2} k^{2} / m-\hat{T}-\varepsilon(B)} .
\end{aligned}
$$

We further define the background Green's function $G_{\mathrm{bg}}^{(+)}\left(k^{2}\right)$ and the closed channel Green's function $G_{\mathrm{cl}}$ as

$$
\begin{aligned}
G_{\mathrm{bg}}^{(+)}\left(k^{2}\right) & =\frac{1}{\hbar^{2} k^{2} / m+i 0^{+}-\hat{T}-V^{(\mathrm{bg})}} \\
G_{\mathrm{cl}}\left(k^{2}\right) & =\frac{1}{\hbar^{2} k^{2} / m-\hat{T}-V^{(\mathrm{cl})}-\varepsilon(B)} .
\end{aligned}
$$

Then we have the relationships

$$
\begin{aligned}
& G_{0}^{(+)}\left(k^{2}\right)=G_{\mathrm{bg}}^{(+)}\left(k^{2}\right)-G_{\mathrm{bg}}^{(+)}\left(k^{2}\right) V^{(\mathrm{bg})} G_{0}^{(+)}\left(k^{2}\right) \\
& G_{0}^{(\mathrm{cl})}\left(k^{2}\right)=G_{\mathrm{cl}}\left(k^{2}\right)-G_{\mathrm{cl}}\left(k^{2}\right) V^{(\mathrm{cl})} G_{0}^{(\mathrm{cl})}\left(k^{2}\right) .
\end{aligned}
$$

Substituting Eqs. (A7) and (A8) into the last terms of the right hand side (r.h.s.) of Eqs. (A1) and (A2), and using the Lippmman Schwinger equation (15) for the background scattering state $\left|\phi_{\vec{k}}^{(\mathrm{bg}+)}\right\rangle$, we get the equation which relates $\left|\Phi_{\vec{k}}^{(+)}\right\rangle$with the background scattering state $\left|\phi_{\vec{k}}^{(\mathrm{bg}+)}\right\rangle[69]$ :

$$
\begin{aligned}
\left|\phi_{\vec{k}}^{(\mathrm{op}+)}\right\rangle & =\left|\phi_{\vec{k}}^{(\mathrm{bg}+)}\right\rangle+G_{\mathrm{bg}}^{(+)}\left(k^{2}\right) W\left|\phi_{\vec{k}}^{(\mathrm{cl}+)}\right\rangle \\
\left|\phi_{\vec{k}}^{(\mathrm{cl}+)}\right\rangle & =G_{\mathrm{cl}}\left(k^{2}\right) W\left|\phi_{\vec{k}}^{(\mathrm{op}+)}\right\rangle .
\end{aligned}
$$


To calculate the p-wave phase shifts $\delta_{1 m_{z}}(k)$, we operate the projection operator $\mathcal{P}_{m_{z}}$ for the manifold $\left(l=1, L_{z}=m_{z}\right)$ on both of the two sides of Eqs. (A9) and (A10). Then we have

$$
\begin{aligned}
& \mathcal{P}_{m_{z}}\left|\phi_{\vec{k}}^{(\mathrm{op}+)}\right\rangle=\mathcal{P}_{m_{z}}\left|\phi_{\vec{k}}^{(\mathrm{bg}+)}\right\rangle+\mathcal{P}_{m_{z}} G_{\mathrm{bg}}^{(+)}\left(k^{2}\right) W \mathcal{P}_{m_{z}}\left|\phi_{\vec{k}}^{(\mathrm{cl}+)}\right\rangle \\
& \mathcal{P}_{m_{z}}\left|\phi_{\vec{k}}^{(\mathrm{cl}+)}\right\rangle=\left|\phi_{\mathrm{res}}^{\left(m_{z}\right)}\right\rangle \frac{\left\langle\phi_{\mathrm{res}}^{\left(m_{z}\right)}\left|W \mathcal{P}_{m_{z}}\right| \phi_{\vec{k}}^{(\mathrm{op}+)}\right\rangle}{\hbar^{2} k^{2} / m-\mu_{\mathrm{res}}\left(B-B_{\mathrm{res}}^{\left(m_{z}\right)}\right)} .(\mathrm{A} 12)
\end{aligned}
$$

Here we have used

$$
\begin{aligned}
\mathcal{P}_{m_{z}} G_{\mathrm{bg}}^{(+)}\left(k^{2}\right) & =\mathcal{P}_{m_{z}} G_{\mathrm{bg}}^{(+)}\left(k^{2}\right) \mathcal{P}_{m_{z}} \\
\mathcal{P}_{m_{z}} G_{\mathrm{cl}}\left(k^{2}\right) & =\mathcal{P}_{m_{z}} G_{\mathrm{cl}}\left(k^{2}\right) \mathcal{P}_{m_{z}}
\end{aligned}
$$

which are guaranteed by the rotational symmetry along the $z$-axis of the system. We also made the approximation

$$
G_{\mathrm{cl}}\left(k^{2}\right) \approx \sum_{m_{z}} \frac{\left|\phi_{\mathrm{res}}^{\left(m_{z}\right)}\right\rangle\left\langle\phi_{\mathrm{res}}^{\left(m_{z}\right)}\right|}{\hbar^{2} k^{2} / m-\mu_{\mathrm{res}}\left(B-B_{\mathrm{res}}^{\left(m_{z}\right)}\right)} .
$$

That is, we only take into account the contribution from the near-resonance bound state $\left|\phi_{\mathrm{res}}^{\left(m_{z}\right)}\right\rangle$ in the closed channel.
Substituting Eqs. (A12) into Eq. (A11), we get

$$
\begin{aligned}
\mathcal{P}_{m_{z}}\left|\phi_{\vec{k}}^{(\mathrm{op}+)}\right\rangle= & \mathcal{P}_{m_{z}}\left|\phi_{\vec{k}}^{(\mathrm{bg}+)}\right\rangle \\
& +G_{\mathrm{bg}}^{(+)}\left(k^{2}\right) W\left|\phi_{\mathrm{res}}^{\left(m_{z}\right)}\right\rangle A^{\left(m_{z}\right)}\left(B, k^{2}\right)
\end{aligned}
$$

with

$$
A^{\left(m_{z}\right)}\left(B, k^{2}\right)=\frac{\left\langle\phi_{\mathrm{res}}^{\left(m_{z}\right)}\left|W \mathcal{P}_{m_{z}}\right| \phi_{\vec{k}}^{(\mathrm{op}+)}\right\rangle}{\hbar^{2} k^{2} / m-\mu_{\mathrm{res}}\left(B-B_{\mathrm{res}}^{\left(m_{z}\right)}\right)} .
$$

Replacing the $\mathcal{P}_{m_{z}}\left|\phi_{\vec{k}}^{(\mathrm{op}+)}\right\rangle$ in the r.h.s. of Eq. (A17) with the r.h.s. of (A16), we get

$$
\begin{aligned}
& A^{\left(m_{z}\right)}\left(B, k^{2}\right) \\
= & \frac{\left\langle\phi_{\mathrm{res}}^{\left(m_{z}\right)}|W| \phi_{\vec{k}}^{(\mathrm{bg}+)}\right\rangle}{\hbar^{2} k^{2} / m-\mu_{\mathrm{res}}\left(B-B_{\mathrm{res}}^{\left(m_{z}\right)}\right)-\left\langle\phi_{\mathrm{res}}^{\left(m_{z}\right)}\left|W G_{\mathrm{bg}}^{(+)}(E) W\right| \phi_{\mathrm{res}}^{\left(m_{z}\right)}\right\rangle} .
\end{aligned}
$$

Substituting Eq. (A18) into Eq. (A16), and using the asymptotic expression (7) of the scattering state and the definition (8) of the partial wave scattering amplitude, we can obtain the p-wave scattering amplitude

$$
f_{1 m_{z}}(k)=f_{1 m_{z}}^{(\mathrm{bg})}(k)-\frac{\pi}{k} \frac{\left\langle\psi_{k 1 m_{z}}^{(\mathrm{bg}-)}|W| \phi_{\mathrm{res}}^{\left(m_{z}\right)}\right\rangle\left\langle\phi_{\mathrm{res}}^{\left(m_{z}\right)}|W| \psi_{k 1 m_{z}}^{(\mathrm{bg}+)}\right\rangle}{\hbar^{2} k^{2} / m-\mu_{\mathrm{res}}\left(B-B_{\mathrm{res}}^{\left(m_{z}\right)}\right)-\left\langle\phi_{\mathrm{res}}^{\left(m_{z}\right)}\left|W G_{\mathrm{bg}}^{(+)}\left(k^{2}\right) W\right| \phi_{\mathrm{res}}^{\left(m_{z}\right)}\right\rangle} .
$$
Here $\left|\psi_{k 1 m_{z}}^{(\mathrm{bg}+)}\right\rangle$ is defined in Eq. (12). $\left|\psi_{k 1 m_{z}}^{(\mathrm{bg}-)}\right\rangle$ is defined as
as

$$
\left|\phi_{\vec{k}}^{(\mathrm{bg}-)}\right\rangle=\left(\frac{2}{m \hbar k}\right)^{\frac{1}{2}} \sum_{l, m_{z}}\left|\psi_{k 1 m_{z}}^{(\mathrm{bg}-)}\right\rangle Y_{l}^{m_{z}}(\hat{k})^{*} .
$$

with

$$
\left|\phi_{\vec{k}}^{(\mathrm{bg}-)}\right\rangle=|\vec{k}\rangle+\frac{1}{\hbar^{2} k^{2} / m+i 0^{-}-\hat{T}-V^{(\mathrm{bg})}} V^{(\mathrm{bg})}|\vec{k}\rangle
$$

the background state with in-going boundary condition. In the above calculation we also used the asymptotic behavior of the background Green's function:

$$
\lim _{r \rightarrow \infty}\left\langle\vec{r}\left|G_{\mathrm{bg}}^{(+)}\left(k^{2}\right)\right| \vec{r}^{\prime}\right\rangle=-m \sqrt{\frac{\pi}{2 \hbar}} \frac{e^{i k r}}{r}\left\langle\phi_{k \hat{r}}^{(\mathrm{bg}-)} \mid \vec{r}\right\rangle
$$

with $\hat{r}=\vec{r} / r$.

With straightforward calculation, we can further rewrite the scattering amplitude $f_{1 m_{z}}(k)$ in Eq. A19)

with

$$
\begin{aligned}
& \mathcal{C}(k)=\frac{k}{\pi} \times \frac{1}{\left|\left\langle\phi_{\mathrm{res}}^{\left(m_{z}\right)}|W| \psi_{k 10}^{(+)}\right\rangle\right|^{2}} \times \\
& \left(\hbar^{2} k^{2} / m-\mu_{\mathrm{res}}\left(B-B_{\mathrm{res}}^{\left(m_{z}\right)}\right)-g_{m_{z}}\left(k^{2}\right)\right) .
\end{aligned}
$$

In the derivation of Eq. A23) we have used the relationship (Appendix B)

$$
\left|\psi_{k 1 m_{z}}^{(-)}\right\rangle=e^{-2 i \delta_{1}^{(\mathrm{bg})}(k)}\left|\psi_{k 1 m_{z}}^{(+)}\right\rangle
$$


and

$$
\begin{aligned}
G_{\mathrm{bg}}^{(+)}\left(k^{2}\right)= & (m \hbar) \int d \overrightarrow{k^{\prime}} \frac{\left|\phi_{k^{\prime}}^{(\mathrm{bg}+)}\right\rangle\left\langle\phi_{\overrightarrow{k^{\prime}}}^{(\mathrm{bg}+)}\right|}{k^{2}+i 0^{+}-k^{\prime 2}} \\
= & -\left(m^{2} / \hbar\right) \pi i \int d \overrightarrow{k^{\prime}} \delta\left(k^{2}-k^{\prime 2}\right)\left|\phi_{\overrightarrow{k^{\prime}}}^{(\mathrm{bg}+)}\right\rangle\left\langle\phi_{\overrightarrow{k^{\prime}}}^{(\mathrm{bg}+)}\right| \\
& +(m \hbar) \mathrm{P} \int d \overrightarrow{k^{\prime}} \frac{\left|\phi_{\overrightarrow{k^{\prime}}}^{(\mathrm{bg}+)}\right\rangle\left\langle\phi_{\overrightarrow{k^{\prime}}}^{(\mathrm{bg}+)}\right|}{k^{2}-k^{\prime 2}}
\end{aligned}
$$

Here P $\int$ refers to the principle value of the integral.

Considering the relationship (9) between the scattering amplitude $f_{1 m_{z}}(k)$ and the phase shift $\delta_{1 m_{z}}(k)$, it is easy to prove that the phase shift $\delta_{1 m_{z}}(k)$ corresponding to the scattering amplitude (A23) is the one given in Eqs. (10) and (11). It is pointed out that, this result can also be proved with the method in Ref. ([67]).

\section{Appendix B: The scattering states with ingoing and outgoing boundary conditions}

In this appendix we prove the formula $\mathrm{A25}$ in Appendix A. We begin from the relationship [69] between the three dimensional scattering states with ingoing and outgoing boundary conditions:

$$
\left\langle\vec{r} \mid \phi_{\vec{k}}^{(\mathrm{bg}-)}\right\rangle=\left\langle\phi_{-\vec{k}}^{(\mathrm{bg}+)} \mid \vec{r}\right\rangle .
$$

Considering the definitions (12) and (1. 20$)$ of $\left|\psi_{k l m_{z}}^{(\mathrm{bg} \pm)}\right\rangle$, we can obtain

$$
\sum_{l, m_{z}}\left\langle\vec{r} \mid \psi_{k l m_{z}}^{(\mathrm{bg}+)}\right\rangle Y_{l}^{m_{z}}(\hat{k})^{*}=\sum_{l, m_{z}}\left\langle\psi_{k l m_{z}}^{(\mathrm{bg}-)} \mid \vec{r}\right\rangle Y_{l}^{m_{z}}(-\hat{k})
$$

We further define the one dimensional functions $F_{k 1}^{(\mathrm{bg} \pm)}(r)$ as [66]

$$
\left\langle\vec{r} \mid \psi_{k 1 m_{z}}^{(\mathrm{bg} \pm)}\right\rangle=i^{l} \frac{1}{\hbar}\left(\frac{m}{\pi k}\right)^{\frac{1}{2}} \frac{1}{r} F_{k 1}^{(\mathrm{bg} \pm)}(r) Y_{1}^{m_{z}}(\hat{r}) .
$$

Using the relationships

$$
\begin{aligned}
Y_{1}^{m_{z}}(-\hat{k}) & =(-1)^{m_{z}+1} Y_{1}^{-m_{z}}(\hat{k})^{*} \\
Y_{1}^{m_{z}}(\hat{r}) & =(-1)^{m_{z}} Y_{1}^{-m_{z}}(\hat{r})^{*} .
\end{aligned}
$$

we get

$$
F_{k 1}^{(\mathrm{bg}+)}(r)=F_{k 1}^{(\mathrm{bg}-)}(r)^{*}
$$

On the other hand, we know that $F_{k 1}^{(\mathrm{bg}+)}(r)$ and $F_{k 1}^{(\mathrm{bg}-)}(r)$ satisfy the same differential equation

$$
\left(-\frac{d^{2}}{d r^{2}}+V^{(\mathrm{bg})}+\frac{2}{r^{2}}\right) F_{k 1}^{(\mathrm{bg} \pm)}(r)=k^{2} F_{k 1}^{(\mathrm{bg} \pm)}(r) .
$$

with the same boundary condition $F_{k 1}^{(\mathrm{bg} \pm)}(0)=0$. Then $F_{k 1}^{(\mathrm{bg}-)}(r)$ is proportional to $F_{k 1}^{(\mathrm{bg}+)}(r)$. To calculate the ratio between $F_{k 1}^{(\mathrm{bg} \pm)}(r)$, we consider their asymptotic behaviors in the limit $r \rightarrow \infty$ :

$$
\begin{aligned}
& F_{k 1}^{(\mathrm{bg}+)}(r)=\hat{j}_{1}(k r)+k f_{k 1}^{(\mathrm{bg})}(k)\left[\hat{n}_{1}(k r)+i \hat{j}_{1}(k r)\right] \\
& F_{k 1}^{(\mathrm{bg}-)}(r)=\hat{j}_{1}(k r)+k f_{k 1}^{(\mathrm{bg})}(k)^{*}\left[\hat{n}_{1}(k r)-i \hat{j}_{1}(k r)\right]
\end{aligned}
$$

with $\hat{j}_{1}(x)$ defined in (21) and

$$
\hat{n}_{1}(x)=-\frac{\cos x}{x}-\sin x
$$

the irregular first order Riccati-Bessel function. Comparing the coefficients of $\hat{n}_{l}(k r)$, we have

$$
F_{k 1}^{(\mathrm{bg}-)}(r)=e^{-2 i \delta_{1}^{(\mathrm{bg})}(k)} F_{k 1}^{(\mathrm{bg}-)}(r) .
$$

Here we have used

$$
\begin{aligned}
f_{k 1}^{(\mathrm{bg})}(k) & =-\frac{1}{i k-k \cot \delta_{1}^{(\mathrm{bg})}(k)} \\
& =\frac{1}{k} \sin \delta_{1}^{(\mathrm{bg})}(k) e^{i \delta_{1}^{(\mathrm{bg})}(k)}
\end{aligned}
$$

Substituting Eq. (B11) into Eq. (B3), we get Eq. (A25).

\section{Appendix C: The expansion of the factor $g_{m_{z}}\left(k^{2}\right)$}

In this appendix we prove the Eq. (17). We firstly rewrite the factor $g_{m_{z}}\left(k^{2}\right)$ as

$$
g_{m_{z}}\left(k^{2}\right)=\operatorname{Re}\left\langle\phi_{\mathrm{res}}^{\left(m_{z}\right)}\left|W G_{+}^{(\mathrm{bg})}(0) W\right| \phi_{\mathrm{res}}^{\left(m_{z}\right)}\right\rangle-k^{2} \operatorname{Re}\left\langle\phi_{\mathrm{res}}^{\left(m_{z}\right)}\left|W G_{+}^{(\mathrm{bg})}(0) G_{+}^{(\mathrm{bg})}\left(k^{2}\right) W\right| \phi_{\mathrm{res}}^{\left(m_{z}\right)}\right\rangle .
$$

Here we have used the identity

$$
G_{+}^{(\mathrm{bg})}\left(k^{2}\right)=G_{+}^{(\mathrm{bg})}(0)-k^{2} G_{+}^{(\mathrm{bg})}(0) G_{+}^{(\mathrm{bg})}\left(k^{2}\right) .
$$

The first term in the r.h.s. of (C1) is independent on $k$. It contributes the constant term $g_{m_{z}}^{(0)}$ in Eq. (17). 
On the other hand, the second term in Eq. (C1) can be re-written as

$$
\begin{aligned}
& k^{2} \operatorname{Re}\left\langle\phi_{\mathrm{res}}^{\left(m_{z}\right)}\left|W G_{+}^{(\mathrm{bg})}(0) G_{+}^{(\mathrm{bg})}\left(k^{2}\right) W\right| \phi_{\mathrm{res}}^{\left(m_{z}\right)}\right\rangle \\
= & \frac{m^{2} k^{2}}{\hbar^{4}} \operatorname{Re} \lim _{\varsigma_{1}, \varsigma_{2} \rightarrow 0^{+}} \int d \overrightarrow{k^{\prime}} \frac{\left|\left\langle\phi_{\mathrm{res}}^{\left(m_{z}\right)}|W| \phi_{\overrightarrow{k^{\prime}}}^{(\mathrm{bg}+)}\right\rangle\right|^{2}}{\left(k^{\prime 2}-i \varsigma_{1}\right)\left(k^{\prime 2}-k^{2}-i \varsigma_{2}\right)} .
\end{aligned}
$$

In the limit $k=0$, we have

$$
\begin{aligned}
& \lim _{k \rightarrow 0} \operatorname{Re}\left\langle\phi_{\text {res }}^{\left(m_{z}\right)}\left|W G_{+}^{(\mathrm{bg})}(0) G_{+}^{(\mathrm{bg})}\left(k^{2}\right) W\right| \phi_{\text {res }}^{\left(m_{z}\right)}\right\rangle \\
\propto & \int d p \frac{\left|\left\langle\phi_{\mathrm{res}}^{\left(m_{z}\right)}|W| \psi_{p 1 m_{z}}^{(\mathrm{bg}+)}\right\rangle\right|^{2}}{p^{3}} .
\end{aligned}
$$

We know that the function $\left|\left\langle\phi_{\text {res }}^{\left(m_{z}\right)}|W| \psi_{p 1 m_{z}}^{(\mathrm{bg}+)}\right\rangle\right|^{2}$ decays to zero when $p \rightarrow \infty$. On the other hand, as we have shown in Sec. III.B, the factor $|\mathscr{J}(k)|^{2}$ tends to a nonzero constant in the low energy limit if the background scattering volume in the open channel is finite. Using the relationship (18) between $\mathscr{J}(k), \tilde{F}_{k 1}^{(\mathrm{bg} \pm)}(r)$ and $\left|\psi_{p 1 m_{z}}^{(\mathrm{bg}+)}\right\rangle$, and the low-energy behavior (22) of $\tilde{F}_{k 1}^{(\mathrm{bg} \pm)}(r)$, it is easy to prove that the factor $\left|\left\langle\phi_{\text {res }}^{\left(m_{z}\right)}|W| \psi_{p 1 m_{z}}^{(\mathrm{bg}+)}\right\rangle\right|^{2}$ is proportional to $p^{3}$ in the limit $p \rightarrow 0$. Therefore, the above integration in Eq. C4 converges to a finite constant in the limit $k^{2} \rightarrow 0$. Then the expansion in Eq. (17) is proved and we have

$$
g_{m_{z}}^{(2)}=-\left\langle\phi_{\mathrm{res}}^{\left(m_{z}\right)}\left|W G_{+}^{(\mathrm{bg})}(0) G_{+}^{(\mathrm{bg})}(0) W\right| \phi_{\mathrm{res}}^{\left(m_{z}\right)}\right\rangle \leq 0 .
$$

\section{Appendix D: The background Jost function}

In this appendix we calculate the Jost function $\mathscr{J}(k)$ of the background scattering state. To this end, we introduce a function $\bar{F}_{k 1}^{(\mathrm{bg} \pm)}(r)=\tilde{F}_{k 1}^{(\mathrm{bg} \pm)}(r) / k^{2}$ where $\tilde{F}_{k 1}^{(\mathrm{bg} \pm)}(r)$ is defined in Eq. (18). It is apparent that $\bar{F}_{k 1}^{(\mathrm{bg} \pm)}(r)$ is a solution of the radial equation

$$
\left(-\frac{d^{2}}{d r^{2}}+V^{(\mathrm{bg})}(r)+\frac{2}{r^{2}}\right) \bar{F}_{k 1}^{(\mathrm{bg})}(r)=k^{2} \bar{F}_{k 1}^{(\mathrm{bg})}(r)
$$

with a $k$-independent boundary condition

$$
\bar{F}_{k 1}^{(\mathrm{bg})}(r \rightarrow 0) \rightarrow r^{2}
$$

Following the spirit of quantum defect theory [4], we assume the scattering potential $V^{(\mathrm{bg})}(r)$ can be approximated as the van der Waals potential $-\hbar^{2} \beta_{6}^{4} /\left(r^{6} m\right)$ when $r$ is larger than a critical distance $r_{0}$ which is much smaller than $\beta_{6}$. In the region with $r<r_{0}, V^{(\mathrm{bg})}(r)$ is assumed to be so large that the atomic kinetic energy $k^{2}$ is negligible in comparing with $V^{(\mathrm{bg})}(r)$, and then $\bar{F}_{k 1}^{(\mathrm{bg})}(r)$ is independent on $k$.

In the region $r>r_{0}, \bar{F}_{k 1}^{(\mathrm{bg})}(r)$ is the superposition of the two independent solutions $\chi_{\epsilon 1}^{(0)}(r)$ and $\kappa_{\epsilon 1}^{(0)}(r)$ of Eq. (D1) [68] (in Ref. [68], $\chi_{\epsilon 1}^{(0)}(r)$ and $\kappa_{\epsilon 1}^{(0)}(r)$ are denoted as $f_{\epsilon 1}^{(0)}(r)$ and $\left.g_{\epsilon 1}^{(0)}(r)\right)$ :

$$
\bar{F}_{k 1}^{(\mathrm{bg})}(r)=\alpha_{k} \chi_{k^{2} 1}^{(0)}(r)+\beta_{k} \kappa_{k^{2} 1}^{(0)}(r)
$$

In the short distance region with $r<<\beta_{6}, \chi_{k^{2} 1}^{(0)}(r)$ and $\kappa_{k^{2} 1}^{(0)}(r)$ are almost independent on $k[74]$.

The wave function $\bar{F}_{k 1}^{(\mathrm{bg})}(r)$ in the two regions are connected at point $r=r_{0}$, where $\bar{F}_{k 1}^{(\mathrm{bg})}(r), \chi_{\epsilon 1}^{(0)}(r)$ and $\kappa_{\epsilon 1}^{(0)}(r)$ are approximately independent on $k$. Then we know that $\alpha_{k}$ and $\beta_{k}$ are independent on $k$ and we have

$$
\bar{F}_{k 1}^{(\mathrm{bg})}(r)=\alpha \chi_{\epsilon_{k} 1}^{(0)}(r)+\beta \kappa_{\epsilon_{k} 1}^{(0)}(r)
$$

with $\epsilon_{k}=\hbar^{2} k^{2} / m$. The above equation yields

$$
\tilde{F}_{k 1}^{(\mathrm{bg})}(r)=k^{2} \alpha \chi_{\epsilon_{k} 1}^{(0)}(r)+k^{2} \beta \kappa_{\epsilon_{k} 1}^{(0)}(r) .
$$

In the region $r \rightarrow \infty$, the asymptotic behaviors of $\chi_{\epsilon_{k} 1}^{(0)}(r)$ and $\kappa_{\epsilon_{k} 1}^{(0)}(r)$ can be expressed in terms of $Z_{i j}(k)$ $(i, j=f, g)$ defined in [68]

$\chi_{\epsilon_{k} 1}^{(0)}(r) \rightarrow \sqrt{\frac{2}{\pi k}}\left[Z_{f f} \sin \left(k r-\frac{\pi}{2}\right)-Z_{f g} \cos \left(k r-\frac{\pi}{2}\right)\right] ;$

$\kappa_{\epsilon_{k} 1}^{(0)}(r) \rightarrow \sqrt{\frac{2}{\pi k}}\left[Z_{g f} \sin \left(k r-\frac{\pi}{2}\right)-Z_{g g} \cos \left(k r-\frac{\pi}{2}\right)\right]$.

On the other hand we know that in the same limit we have 66

$$
\tilde{F}_{k 1}^{(\mathrm{bg})}(r)=\mathscr{J}(k)\left[e^{i \delta_{1}^{(\mathrm{bg})}(k)} \sin \left(k r-\frac{\pi}{2}+\delta_{1}^{(\mathrm{bg})}(k)\right)\right]
$$

Together with Eqs. (D5), (D6), (D7) as well as (D8), we obtain the expression of (34) of $|\mathscr{J}(k)|^{2}$ and the background phase shift:

$$
\tan \delta_{1}^{(\mathrm{bg})}=-\frac{K_{l=1}^{0} Z_{g g}-Z_{f g}}{K_{l=1}^{0} Z_{g f}-Z_{f f}}
$$

where $D_{i j}(k)=\left(k \beta_{6}\right)^{3 / 2} Z_{i j}(k)$ and $K_{l=1}^{0}=-\beta / \alpha$ is the one in Sec. III. The result (D9) is also given in [2]. 
[3] C. A. Regal, C. Ticknor, J.L. Bohn, and D.S. Jin. Phys. Rev. Lett. 90, 053201 (2003).

[4] Kenneth Günter, Thilo Stöferle, Henning Moritz, Michael Köhl, and Tilman Esslinger, Phys. Rev. Lett. 95, 230401 (2005).

[5] C. Ticknor, C. A. Regal, D. S. Jin, and J. L. Bohn, Phys. Rev. A 69, 042712 (2004).

[6] C. A. Regal, C. Ticknor, J. L. Bohn, and D. S. Jin, Nature 424, 47 (2003).

[7] J. P. Gaebler, J. T. Stewart, J. L. Bohn, and D. S. Jin, Phys. Rev. Lett. 98, 200403 (2007).

[8] J. Zhang, E. G. M. van Kempen, T. Bourdel, L. Khaykovich, J. Cubizolles, F. Chevy, M. Teichmann, L. Tarruell, S. J. J. M. F. Kokkelmans, and C. Salomon, Phys. Rev. A 70, 030702(R)(2004).

[9] C. H. Schunck, M. W. Zwierlein, C. A. Stan, S. M. F. Raupach, W. Ketterle, A. Simoni, E. Tiesinga, C. J. Williams, and P. S. Julienne, Phys. Rev. A 71, 045601 (2005).

[10] R. A. W. Maier, C. Marzok, and C. Zimmermann and Ph. W. Courteille, Phys. Rev. A 81, 064701 (2010).

[11] J. Fuchs, C. Ticknor, P. Dyke, G. Veeravalli, E. Kuhnle, W. Rowlands, P. Hannaford, and C. J. Vale, Phys. Rev. A 77, 053616 (2008).

[12] Y. Inada, M. Horikoshi, S. Nakajima, M. KuwataGonogami, M. Ueda, and T. Mukaiyama, Phys. Rev. Lett. 101, 100401 (2008).

[13] B. Deh, C. Marzok, C. Zimmermann, and Ph. W. Courteille, Phys. Rev. A 77, 010701(R) (2008).

[14] E. Wille, F. M. Spiegelhalder, G. Kerner, D. Naik, A. Trenkwalder, G. Hendl, F. Schreck, R. Grimm, T. G. Tiecke, J. T. M. Walraven, S. J. J. M. F. Kokkelmans, E. Tiesinga, and P. S. Julienne, Phys. Rev. Lett. 100, 053201 (2008).

[15] F. Ferlaino et. al., Phys. Rev. A 73040702 (2006).

[16] L. You and M. Marinescu, Phys. Rev. A, 60, 2324 (1999).

[17] S. S. Botelho, C. A. R. SódeMelo, arXiv: condmat/0409357.

[18] F. R. Klinkhamer and G. E. Volovik, JETP Lett. 80, 389 (2004).

[19] S.S. Botelho, C. A. R. SódeMelo, J. Low. Temp. Phys. 140, 409 (2005).

[20] Y. Ohashi, Phys. Rev. Lett. 94, 050403 (2005).

[21] M. Iskin and C. A. R. SódeMelo, Phys. Rev. B 72, 224513 (2005).

[22] W. Vincent Liu, Phys. Rev. A 72, 053613 (2005).

[23] M. Iskin and C. A. R. SódeMelo, Phys. Rev. Lett. 96, 040402 (2006).

[24] Chi-Ho Cheng and Sung-Kit Yip, Phys. Rev. B 73, 064517 (2006).

[25] Eytan Grosfeld, Nigel R. Cooper, Ady Stern, and Roni Ilan, Phys. Rev. B 76, 104516 (2007).

[26] R. Liao, F. Popescu, K. Quader, arXiv: 0711.4594.

[27] Yajiang Hao, Yunbo Zhang, Shu Chen, Phys. Rev. A 76, 063601 (2007).

[28] J. Levinsen, N. Cooper, V. Gurarie, Phys. Rev. Lett. 99, 210402 (2007).

[29] Wei Zhang and C. A. R. SódeMelo, Phys. Rev. A 76, 013627 (2007).

[30] M. D. Girardeau1 and E. M. Wright, Phys. Rev. A 77, 043612 (2008).

[31] K. Quader, R. Liao, and F. Popescu, Int. J. Mod. Phys. B 22, 4358 (2008).

[32] Yasumasa Tsutsumi and Kazushige Machida, J. Phys.
Soc. Jpn. 78, 084702 (2009).

[33] Y.-J. Han, Y.-H. Chan, W. Yi, A. J. Daley, S. Diehl, P. Zoller, and L.-M. Duan, PRL 103, 070404 (2009).

[34] Kazunori Suzuki, Takahiko Miyakawa, and Toru Suzuki, Phys. Rev. A 77, 043629 (2008).

[35] C.-H. Cheng and S.-K. Yip, Phys. Rev. Lett. 95, 070404 (2005).

[36] V. Gurarie, L. Radzihovsky, and A. V. Andreev, Phys. Rev. Lett. 94, 230403(2005).

[37] T.-L. Ho and R. B. Diener, Phys. Rev. Lett. 94, 090402 (2005).

[38] V. Gurarie, L. Radzihovsky, Ann. Phys., 322, 2 (2007).

[39] A. del Campo, J. G. Muga, M. D. Girardeau, Phys. Rev. A 76, 013615 (2007).

[40] M. Iskin and C. J. Williams, Phys. Rev. A 77, 041607(R) (2008)

[41] J. Levinsen, N. R. Cooper and V. Gurarie, Phys. Rev. A 78, 063616 (2008).

[42] Leo Radzihovsky and Sungsoo Choi, PRL 103, 095302 (2009).

[43] Y. Tsutsumi, K. Machida, J. Phys. Soc. Jpn. 78, 084702 (2009).

[44] Y. Tsutsumi, K. Machida, J. Phys. Soc. Jpn. 79, 034301 (2010).

[45] N. T. Zinner, Eur. Phys. J. D 57, 235 (2010).

[46] T. Mizushima, M. Ichioka, and K. Machida, PRL 101, 150409 (2008).

[47] M. D. Girardeau1, and G. E. Astrakharchik, Phys. Rev. A 81, 043601 (2010).

[48] T. Mizushima and K. Machida, Phys. Rev. A 82, 023624 (2010).

[49] T. Mizushima and K. Machida, Phys. Rev. A 81, 053605 (2010).

[50] T. Mizushima and K. Machida, Phys. Rev. A 80, 035601 (2009).

[51] G. Moller, N. R. Cooper and V. Gurarie, arXiv:1006.0924

[52] K. B. Gubbels and H. T. C. Stoof, Phys. Rev. Lett. 99, 190406 (2007).

[53] Frédéric Chevy, E. G. M. Van Kempen, T. Bourdel, J. Zhang, L. Khaykovich, M. Teichmann, L. Tarruell, S. J. J. M. F. Kokkelmans and C. Salomon, Phys. Rev. A, 71, 062710 (2005).

[54] S.-K. Yip, Phys. Rev. A 78, 013612 (2008).

[55] Mattia Jona-Lasinio, Ludovic Pricoupenko, Yvan Castin, Phys. Rev. A 77, 043611 (2008).

[56] Ludovic Pricoupenko, Phys. Rev. Lett, 96, 050401 (2006).

[57] R. Roth and H. Feldmeier Phys. Rev. A, 64, 043603 (2001).

[58] Zbigniew Idziaszek and Tommaso Calarco, Phys. Rev. Lett. 96, 013201 (2006).

[59] RenéStock, Andrew Silberfarb, Eric L. Bolda, and Ivan H. Deutsch, Phys. Rev. Lett. 94, 023202 (2005).

[60] J. H. Macek and J. Sternberg, Phys. Rev. Lett. 97, 023201 (2006).

[61] Brian E. Granger and D. Blume, Phys. Rev. Lett. 92, 133202 (2004).

[62] M. Lysebo and L. Veseth, Phys. Rev. A 79, 062704 (2009).

[63] Z. Li and K. W. Madison, Phys. Rev. A 79, 042711 (2009).

[64] Akira Suzuki,Yi Liang and Rajat K. Bhaduri, Phys. Rev. A 80, 033601 (2009). 
[65] Zbigniew Idziaszek Phys. Rev. A 79, 062701 (2009).

[66] J. R. Taylor, Scattering Theory, Wiley, New York, 1972.

[67] U. Fano, Phys. Rev. 124, 1866 (1961).

[68] B. Gao, Phys. Rev. A 58, 1728 (1998).

[69] T. Kohler, K. Goral and P. S. Julienne, Rev. Mod. Phys. 78, 1311 (2006).

[70] Bo Gao, Phys. Rev. A 80, 012702 (2009).

[71] J. L. Bohn, Phys. Rev. A 61, 053409 (2000).

[72] M. Jona-Lasinio, L. Pricoupenko, and Y. Castin, Phys. Rev. 77, 043611 (2008).
[73] F. Chevy, E. G. M. van Kempen, T. Bourdel, J. Zhang, L. Khaykovich, M. Teichmann, L. Tarruell, S. J. J. M. F. Kokkelmans, and C. Salomon, Phys. Rev. A 71, 062710 (2005).

[74] B. Gao, Eur. Phys. J. D 31, 283 (2004).

[75] A. Derevianko, et. al., Phys. Rev. Lett. 82, 3589 (1999).

[76] Z.-C. Yan, J. F. Babb, A. Dalgarno, and G. W. F. Drake, Phys. Rev. A 54, 2824 (1996). 